\title{
Charged State of a Spherical Plasma in Vacuum
}

\author{
F. Cornolti, ${ }^{1}$ F. Ceccherini, ${ }^{1,}$ \& S. Betti, ${ }^{1}$ and F. Pegoraro ${ }^{1}$ \\ ${ }^{1}$ Dipartimento di Fisica "Enrico Fermi" 8 INFM, \\ Università di Pisa, Largo B. Pontecorvo 3, 56127 Pisa, Italy
}

\begin{abstract}
The stationary state of a spherically symmetric plasma configuration is investigated in the limit of immobile ions and weak collisions. Configurations with small radii are positively charged as a significant fraction of the electron population evaporates during the equilibration process, leaving behind an electron distribution function with an energy cutoff. Such charged plasma configurations are of interest for the study of Coulomb explosions and ion acceleration from small clusters irradiated by ultraintense laser pulses and for the investigation of ion bunches propagation in a plasma.
\end{abstract}

PACS numbers:

\section{INTRODUCTION}

The interaction of ultraintense laser pulses with solid targets leads to the formation of plasmas with unusual properties in terms of particle energy distributions and of spatial mass and charge density distributions. Such properties can be exploited in order to obtain sources of high energy electromagnetic radiation and charged particle beams with unprecedented intensities and time and space resolutions. For the intensities of present day ultrashort, superintense laser pulses, the energy that the ions in a target acquire due to direct interaction with the electromagnetic fields of the laser pulse is usually small, while the energy of the plasma electrons can be of the order of tens of KeV. These "hot" electrons expand until their "pressure" is balanced by the electrostatic field that sets up due to spatial charge separation [1, 2, 3]. This process leads to a steady state configuration (SSC) which is reached in a time of the order of some electron plasma periods. Thus, since the ion response time is much longer than that of the electrons, SSC can be achieved before the ions can depart significantly from their initial configuration. Afterwards ion acceleration takes place, as predicted theoretically 1, 2, 3, , 4, 5, 6, 7, 8, 9, 10, 11, 12, 13, 14, and confirmed experimentally 15, 16, 17, 18, 19, 20, 21, 22, 23, 24, 25, 26]. Clearly, this description does not apply to highly relativistic regimes like those described in 27].

The aim of this paper is to present a combined analytical and numerical investigation of three dimensional SSC characterized by a hot electron plasma and a cold (immobile) ion core. Such configurations are especially appealing because, contrary to one dimensional geometries, in three dimensional cases charged SSC are expected to set up. In fact, while in the former case an infinite energy is required in order to bring a charge to infinity, in the latter the energy necessary for electron evaporation is finite. In particular, we show that the SSC charging up and the energy distribution of the

*Electronic address: ceccherini@df.unipi.it electrons depend on the ratio between the radius of the ion core and the electron Debye length and on the history of the electron expansion. The understanding of the SSC charging up with immobile ions and of the electron energy distribution is relevant to many experimental conditions as these processes affect the way in which ions are accelerated on longer time scales when the constraint of a fixed ion core is removed. In the case of the Coulomb explosion of a small cluster 28, 29, 30, 31, 32, 33, 34] these processes affect the value of the maximum energy that the ions can acquire in the acceleration process. Furthermore, in applications related to proton imaging 39, 40, 41 and to the propagation of ion beams in solid targets 42, the Coulomb repulsion and the screening effect of the electrons can strongly affect the proton trajectories. This is also the case for applications of proton laser acceleration to hadron therapy [43, 44, 45, 46, 47, 48, 49, 50, where a very precise collimation of the proton beam and a high energy resolution are essential. Moreover, as discussed in [52], the topic of the charging of a spherical plasma in less extreme conditions can play a key role in the study of dusty plasmas [53], and aerosols [54].

The paper is organized as follows. In Sec.III simple relationships are derived analytically on the basis of two schematic models that are introduced in order to grasp the main features of the plasma charging process in different collisionality regimes. In Sec.III] we present the results of a series of numerical simulations obtained with a one-dimensional Particle in cell code (PIC) in a spherical geometry and with fixed ions. We then compare the numerically obtained charge values and electron energy distributions with those obtained from the analytical models. Finally, conclusions are presented in Sec.IV]

\section{SIMPLIFIED MODELS OF THE CHARGING PROCESS}

In this section we discuss two simplified models that allow us to identify the main physical features of the plasma charging process. These models rely on assigning a simplified condition for the electrons to leave the ion core 
and on two different rules for the energy redistribution of the remaining electrons.

As a starting configuration we assume the following electron and ion density profiles

$$
n_{e}(r)=n_{i}(r)=n_{0} \theta(1-r / R),
$$

with $\theta(x)=1$ for $x>0$ and $\theta(x)=0$ for $x<0$. Here $R$ is the radius of the ion plasma core and $N_{0}=n_{0}\left(4 \pi R^{3} / 3\right)$ the ion and electron initial particle number. We denote by $N_{e}$ the time dependent number of electrons inside the ion core.

Initially electrons have a Maxwellian energy distribution with temperature $T_{0}$. Moreover, in these models the electron density is taken to be uniform inside the ion core. As a further simplification, we assume that, on average, the radial crossing of the electron trajectories does not lead to a relative redistribution of the charge in front and behind each electron outside the core. This simplification allows us to assume that, after leaving the ion core each electron moves as if in an effective time independent Coulomb field. Hence, the condition for an electron to reach infinity is that it has a positive total energy when it reaches the ion core surface at $r=R$. In this model the particles which satisfy this condition are said to "evaporate" and are assumed to be lost when they reach $r=R$. On the contrary, the electrons that have a negative energy at $r=R$ are assumed to remain inside the ion core. Furthermore, we assume that inside the ion core the electrons move as free particles. The evaporation of the electrons with positive total energy at $r=R$ changes the number of electrons $N_{e}$, the total energy of the system inside the ion core and causes an energy redistribution of the remaining electrons.

We discuss the "collisional" regime and the "collisionless" one. In the first one, the electrons which are not evaporated thermalize at a temperature $T$, which turns out to be a decreasing function of time. In the second regime no thermalization occurs, and the evaporation causes a progressive depletion in the high energy tail of the electron distribution function, which remains isotropic in velocity space.

In what follows, lengths will be measured in units of the initial Debye length $\lambda_{d}=\left(T_{0} / 4 \pi n_{0} e^{2}\right)^{1 / 2}$, with $e$ the absolute value of the electronic charge, time $t$ in units of $\omega_{p e}^{-1}=\left(4 \pi e^{2} n_{0} / m_{e}\right)^{-1 / 2}$, with $m_{e}$ the electron mass, energies in units of the initial electron temperature $T_{0}$, velocities in units of the initial electron thermal speed $v_{t h, 0}=\sqrt{T_{0} / m_{e}}$, mass in units of the electron mass and particle numbers in units of $N_{0}$. Since inside the ion core the electron density is taken to be uniform, with the adopted normalization the normalized electron density $n_{e}$ and the normalized total number of electrons $N_{e}$ are numerically equal.

\section{A. Collisional regime}

If the electrons inside the ion core are re-thermalized by collisions, their velocity distribution function remains Maxwellian and their time dependent kinetic energy is given by $U_{k}=3 N_{e} T / 2$. The electron evaporation rate is obtained by calculating the flux of electrons with positive total energy through the core surface. Defining the time dependent quantity $\phi_{T}=e \Phi_{R} / T=\left(1-N_{e}\right) R^{2} / 3 T$, with $\Phi_{R}$ the electrostatic potential at the ion core surface, the positive total energy condition corresponds to $v^{2} / 2 T \geq$ $\phi_{T}$. Thus we obtain

$$
\frac{d N_{e}}{d t}=-\frac{3}{\sqrt{2 \pi}} \frac{\left(1+\phi_{T}\right)}{\tau} e^{-\phi_{T}} N_{e},
$$

where $\tau=R / \sqrt{T}$ is the electron crossing time inside the ion core. Analogously, the energy flux $\Phi_{U}$ of the evaporating particle can be computed by noting that each evaporating electron carries away the residual en$\operatorname{ergy} v^{2} / 2 T-\phi_{T}$. Then we obtain

$$
\Phi_{U}=\frac{3}{\sqrt{2 \pi}} \frac{\left(2+\phi_{T}\right)}{\tau} e^{-\phi_{T}} N_{e} T .
$$

The total energy of the system can be written as $U=$ $3 N_{e} T / 2+U_{\Phi}$ where $U_{\Phi}$ is the electrostatic energy of the charged configuration which increases as the electrons evaporate as

$$
\frac{d U_{\Phi}}{d t}=-\frac{2}{5} R^{2}\left(1-N_{e}\right) \frac{d N_{e}}{d t} .
$$

Thus, from the total energy balance we obtain for the time change of the kinetic energy of the system

$$
\begin{aligned}
\frac{d\left(3 N_{e} T / 2\right)}{d t} & =-\frac{3}{\sqrt{2 \pi}} \frac{\left(2+\phi_{T}\right)}{\tau} e^{-\phi_{T}} N_{e} T \\
+ & +\frac{2}{5} R^{2}\left(1-N_{e}\right) \frac{d N_{e}}{d t},
\end{aligned}
$$

which, coupled to Eq. (2), gives the time evolution of the temperature $T$.

\section{B. Collisionless regime}

If on the contrary we assume that plasma electrons inside the ion core are not significantly affected by collisions, their distribution function becomes nonMaxwellian. We assume that the electron distribution remains homogeneous in coordinate space and isotropic in velocity space. Thus, denoting by $N_{\mathcal{E}}$ the time dependent number of electrons with kinetic energy (normalized on the initial temperatute $T_{0}$ ) in the interval $[\mathcal{E}, \mathcal{E}+d \mathcal{E}]$, and introducing the time dependent quantity $\phi_{0}=e \Phi_{R}=\frac{1}{3}\left(1-N_{e}\right) R^{2}$, which differs from $\phi_{T}$ in the 
previous by the normalized temperature factor $1 / T$, we obtain

$$
\frac{d N_{\mathcal{E}}}{d t}=-\frac{3}{2 \sqrt{2}} \frac{\sqrt{\mathcal{E}}}{R} \theta\left(\mathcal{E}-\phi_{0}\right) N_{\mathcal{E}}
$$

This implies that the evaporation of the electron population with energy $\mathcal{E}$ stops at a well defined time $t=t_{\mathcal{E}}$, where $t_{\mathcal{E}}$ is such that $\mathcal{E}=\phi_{0}\left(t_{\mathcal{E}}\right)$. Therefore,

$$
\begin{aligned}
N_{\mathcal{E}} & =N_{\mathcal{E}}(0) e^{-t / t_{d}} \quad \text { for } \quad t \leq t_{\mathcal{E}} \\
& =N_{\mathcal{E}}(0) e^{-t_{\mathcal{E}} / t_{d}} \quad \text { for } \quad t>t_{\mathcal{E}}
\end{aligned}
$$

with $t_{d}=(2 R \sqrt{2} / 3 \sqrt{\mathcal{E}})$ the $\mathcal{E}$-dependent decay time and $N_{\mathcal{E}}(0)$ the electron kinetic energy distribution at the initial time $t=0$. We assume the electron velocities at time $t=0$ to be Maxwellian distributed, hence the initial electron kinetic energy distribution $N_{\mathcal{E}}(0)$ is given by

$$
N_{\mathcal{E}}(0)=N_{\mathcal{E}, 0}=\frac{2}{\sqrt{\pi}} e^{-\mathcal{E}} \sqrt{\mathcal{E}} .
$$

The electron number $N_{e}$, and therefore $\phi_{0}$, can thus be calculated performing numerically, at fixed $t$, the integral $N_{e}=\int d \mathcal{E} N_{\mathcal{E}}$. Note that in this collisionless model a rough estimate of the asymptotic electron number could be obtained by approximating the final electron distribution function with the initial one for $\mathcal{E}<\mathcal{E}^{*}$, and with zero for $\mathcal{E}>\mathcal{E}^{*}$. We can then determine the cutoff energy $\mathcal{E}^{*}$ self consistently by equating its value to the electrostatic energy of the configuration with charge $Q\left(\mathcal{E}^{*}\right)=\int_{\mathcal{E}^{*}}^{\infty} N_{\mathcal{E}}(0) d \mathcal{E}$,

$$
\mathcal{E}^{*}=\left[Q\left(\mathcal{E}^{*}\right) R^{2} / 3\right] .
$$

\section{Discussion of the analytical models}

Numerical integration of Eqs. (2) shows that both in the collisionless and in the collisional regime the electron evaporation rate is significantly reduced from its initial value when the quantities $\phi_{T}, \phi_{0}$ become of order unity. This means that, in the collisional regime, the evaporation nearly stops when the electron electrostatic energy, which is an increasing function of time, is of the order of the electron temperature which decreases with time. Whereas in the collisionless case the electron evaporation is significant only until the electron electrostatic energy is of the order of the initial average electron kinetic energy.

The initial evaporation rate, which is obviously the same in both, the collisional and in the collisionless case, is obtained from either Eq. (2) or Eq. (6) which give

$$
\left.\frac{d N_{e}}{d t}\right|_{t=0}=-\frac{3}{\sqrt{2 \pi}} \frac{1}{R} .
$$

A linear approximation of $N_{e}$ is feasible as long as $t \ll t^{*}, \tilde{t}$ where

$$
t^{*}=\sqrt{2 \pi} R / 3
$$

is the initial characteristic evaporation time and $\tilde{t}$ is defined in the two different collisionality regimes by either the condition $\left.\phi_{T}\right|_{\tilde{t}} \sim 1$ or $\left.\phi_{0}\right|_{\tilde{t}} \sim 1$ and which can be roughly evaluated as

$$
\tilde{t}=3 t^{*} / R^{2}
$$

Therefore, if $t^{*}<\tilde{t}$, i.e., for ion core radii satisfying $R \leq \sqrt{3}$, the charging process continues until almost complete depletion of the electron population.

Finally, we note that the time dependent electron energy distribution predicted in the collisionless regime is highly non thermal. The fact that the electron evaporation only occurs for those particles with kinetic energy $\mathcal{E}$ satisfying $\mathcal{E} \geq\left.\phi_{0}\right|_{t}$ causes a depletion of the high energy tail of the electron distribution function, as will be examined in detail in Sec. III

\section{PIC SIMULATIONS AND COMPARISON WITH THE ANALYTICAL RESULTS}

Our PIC simulations are initialized with the electron and ion density profiles $n_{e}, n_{i}$ given by Eq. (1). The initial electron distribution function is Maxwellian with temperature $T_{0}$. At time $t=0$ the electrons are allowed to move. During their expansion, the electrons that reach the border of the simulation box with positive total energy are removed. Therefore, as the total number of electrons decreases with time, the plasma acquires a net positive charge and an electrostatic potential sets up. Finally a stationary state is reached where no more electrons reach the boundary. We denote by $N_{\infty}, n_{\infty}, E_{\infty}, N_{\mathcal{E}, \infty}$ the electron number, the electron density, the electric field profile, and the electron kinetic energy distribution once SSC has been reached. As expected, our simulations show that these quantities depend on the ion core radius $R$.

The results regarding the profile of both the electric field $E_{\infty}$ and of the electron density $n_{\infty}$ for two different ion plasma radii $R$ are presented in Fig.11 As one can see in Fig.1p, the electrons which are outside the ion sphere are confined in a region whose width is of the order of a few Debye lengths.

The numerical results regarding the electron kinetic energy distribution in the stationary state are presented in Fig.2] In the figure, the ratio $N_{\mathcal{E}, \infty} / N_{\mathcal{E}, 0}$, is shown versus the electron kinetic energy $\mathcal{E}$ in semi-logarithmic scale, for several values of the ion plasma radius $R$. These results show that the electron kinetic energy distribution is highly non-thermal. It exhibits a cut in its high energy tail, and the cutoff energy increases with the ion plasma radius $R$.

Regarding the comparison with the semi-infinite, planar case, our results show that both the electric field and the electron density are very similar to those presented in 6] only as long as the ion core radius is greater than several tens of Debye lengths. Since, contrary to 

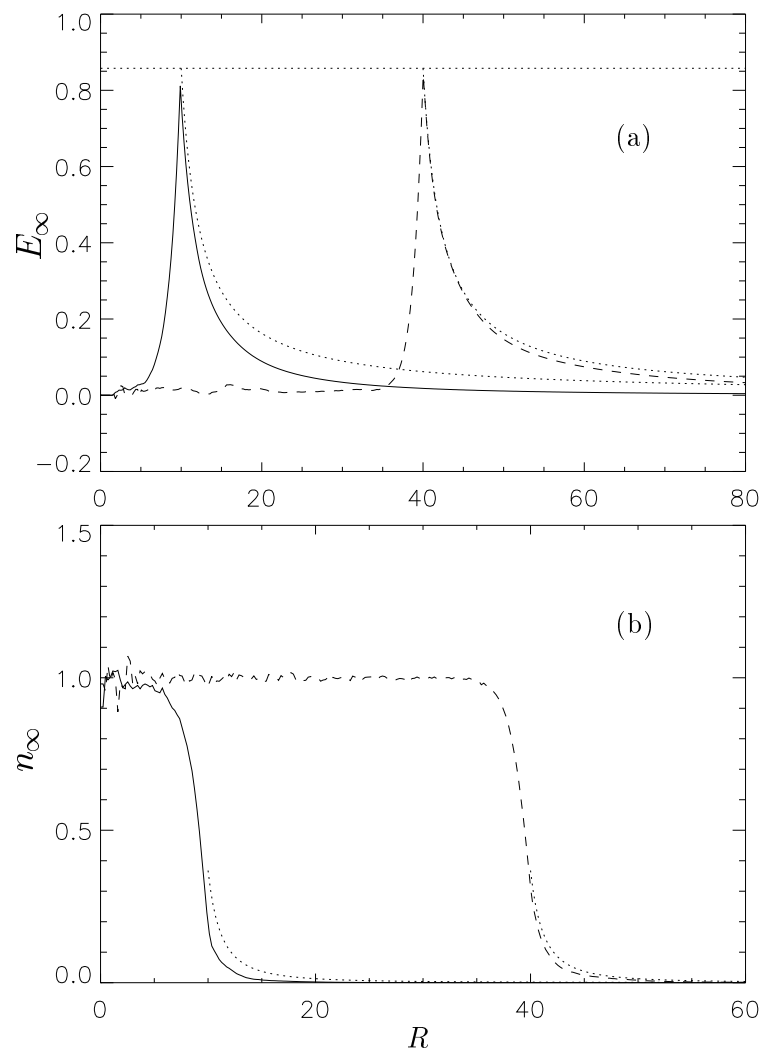

FIG. 1: Spatial profile of the electric field $E_{\infty}$ (panel (a)) and electron density $n_{\infty}$ (panel (b)) for $R=10$ (dashed line) and $R=40$ (solid line). For comparison they are plotted together with the profile that would be obtained in the semiinfinite case (dotted lines) [6]. The straight line in panel (a) corresponds to the maximum value of the electric field in the semi-infinite case, i.e, $E=\sqrt{2 / \mathrm{e}}$.

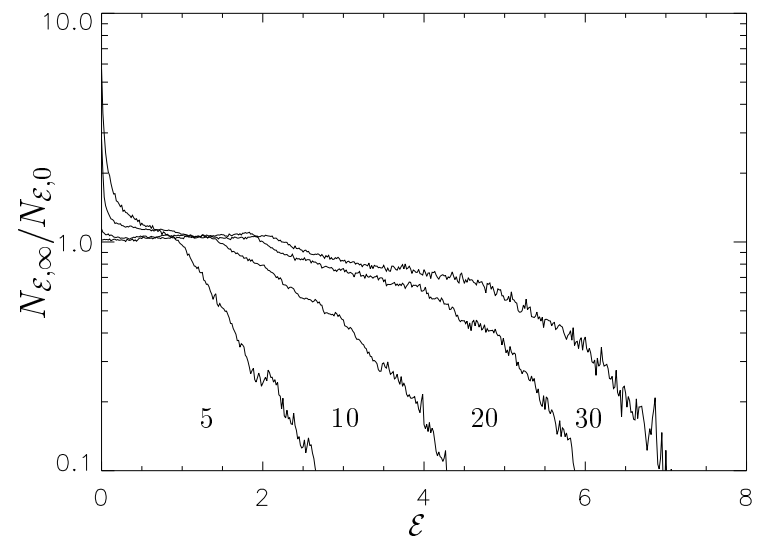

FIG. 2: Behaviour of the ratio between the final and the initial electron kinetic energy distributions for different ion plasma radii.

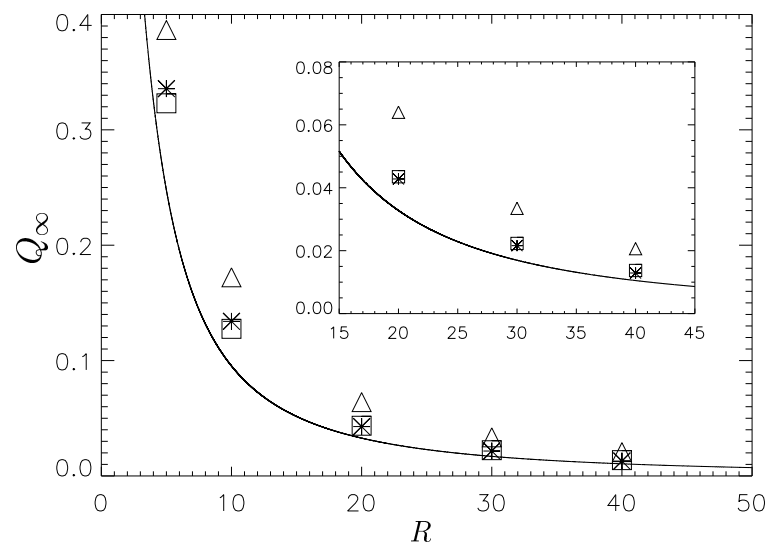

FIG. 3: Comparison between the stationary state value of the dimensionless charge inside the ion core predicted by the collisional model (triangles), the collisionless model (squares), the PIC simulations (stars), and the numerical solution of Eq. (9) (solid line). A magnification of the region $15<R<45$ is also shown in the figure.

a one-dimensional configuration, in the case of a threedimensional configuration the energy required for the electrons to evaporate is finite, the differences observed are mainly due to the cutoff in the electron high energy tail. Such cutoff is responsible for the electron density depletion observed outside the ion core (see Fig. 1b) and, consequently, for the corresponding electric field profile. As shown in Fig. [1 a, in the limit $R \gg 1$ the value of the dimensionless electric field at the surface of the ion core is almost independent of $R$, thus the net dimensionless charge confined within the ion core scales approximately as $1 / R$.

The comparison between the charge value $Q_{\infty}=1-N_{\infty}$ obtained numerically and that predicted analytically is shown in Fig. 3 for different values of the ion core radius $R$. Note that the value of $Q_{\infty}$ obtained numerically includes all SSC electrons, i.e., the electrons inside the ionic sphere and those in the surrounding halo. It is seen that the agreement among the numerical results and the values obtained in the collisionless regime is very good in the whole range $5-40$. With regard to the thermal model adopted for the collisional regime we remark that for small radii it predicts a moderately larger value of $Q_{\infty}$, but the two different regimes lead to very close values $Q_{\infty}$ in case of large radii.

Our results indicate that collisions can affect the charging up process only for small ion core radii. This result can be explained by noting that the potential due to the electron expansion scales as $R^{2}$. Thus, as the potential barrier increases, the fraction of the electron population that, because of Coulomb collisions, reaches a positive total energy and can thus leave the ion core decreases. However, the collisional thermalization of the distribution function assumed in Sec.【A can only be expected 




FIG. 4: Comparison between the cutoff energy $\mathcal{E}_{\text {cut }}$ as a function of the ion core radius $R$ predicted by the collisionless model (squares) and by the PIC simulations (stars).

to to apply when the ion core radius is much larger than the electron mean free path, whereas in most plasma conditions the mean free path due to Coulomb collisions is much greater than the Debye length.

It is worth to note that a precise fit of the numericallyobtained plasma charge state is given by the following Padé approximation

$$
Q_{\infty} \simeq \frac{1+a R}{1+b R+c R^{2}},
$$

with $a=5.6 \cdot 10^{-3}, b=1.4 \cdot 10^{-1}$ and $c=5.5 \cdot 10^{-2}$.

With regard to the high tail of the electron kinetic energy distribution we define the cutoff energy $\mathcal{E}_{\text {cut }}$ as the energy satisfying the relation $\left.\left(N_{\mathcal{E}, \infty} / N_{\mathcal{E}, 0}\right)\right|_{\mathcal{E}=\mathcal{E}_{\text {cut }}}=10^{-1}$. The analytical and numerical results obtained for $\mathcal{E}_{c u t}$ are shown in Fig.4 for different values of the ion core radius $R$. Taking into account these results, we remark that the collisionless model, although not capable of reproducing the fine details of the whole electron kinetic energy distribution observed in the simulations, nevertheless it predicts the cut in the distribution high energy tail with great accuracy.

\section{CONCLUSIONS}

In this paper we have investigated the charging up process of a spherically symmetric plasma configuration in vacuum in the limit of immobile ions. Two different simplified models have been presented. With the help of these models we have established scaling laws relating the steady state total charge and electron energy distribution on the radius of the ion core, normalized in terms of the initial electron Debye length. These scalings have been validated by mean of spherical one-dimensional PIC simulations. The agreement we find is overall very good. Charged plasma configurations such as those investigated in this paper occur naturally in the interaction of ultraintense laser pulses with matter and are of interest, e.g., for setting the initial conditions in the study of Coulomb explosions and ion acceleration from small clusters irradiated by ultraintense laser pulses.

In particular, regarding the problem of cluster expansion the analytical and numerical results that we have presented show that a spherically symmetric configuration of cold ions and hot electrons, which is the typical starting configuration in cluster expansion experiments, does not evolve towards a neutral configuration, in contrast with the one-dimensional planar case. This charging up effect can strongly modify the maximum energy that the ions can gain and the typical timescale on which their acceleration occurs.

\section{Acknowledgments}

This work was supported by the INFM Parallel Computing Initiative. Useful discussions with A. Macchi are gratefully acknowledged.
[1] J. E. Allen and J. G. Andrews, J. Plasma Physics, 4, 187 (1970).

[2] J. E. Crow, P. L. Auer and J. E. Allen, J. Plasma Physics, 14, 65 (1975).

[3] P. D. Prewett and J. E. Allen, J. Plasma Physics, 10, 451 (1973).

[4] T. Nedelea and H. M. Urbassek, Phys. Rev. E 69, 0546408 (2004).

[5] M. Passoni and M. Lontano, Phys. Rev. E 69, 026411 (2004).

[6] P. Mora, Phys. Rev. Lett. 90, 185002 (2003).

[7] A. A. Ivanov and K.S. Serebrennikov, JETP Lett. 78, 123 (2003).

[8] V. F. Kovalev and V. Yu. Bychenkov, Phys. Rev. Lett. 90, 185004 (2003).
[9] V. F. Kovalev, V. Yu. Bychenkov and V. T. Tikhounchuk, JETP Lett. 74, 10 (2001).

[10] D. S. Dorozhkina and V. E. Semenov, Phys. Rev. Lett. 81, 2691 (1998).

[11] D. S. Dorozhkina and V. E. Semenov, JETP Lett. 67, 573 (1998).

[12] A. V. Gurevitch, L. V. Pariskaya and L.P. Pitaievskii, J. Plasma Physics, 14, 65 (1975).

[13] M. Widner, I. Alexeff and W. D. Jones, Phys. Fluids 14, 795 (1971).

[14] S. Betti, F. Ceccherini, F. Cornolti and F. Pegoraro, submitted for publication. Available at http://xxx.lanl.gov/abs/physics/0405030

[15] M. Hegelich et al., Phys. Rev. Lett. 89, 085002 (2002).

[16] J. Badziak et al., Phys. Rev. Lett. 87, 215001 (2001). 
[17] A. J. Mackinnon et al., Phys. Rev. Lett. 86, 1769 (2001).

[18] A. J. Mackinnon et al., Phys. Rev. Lett. 88, 215006 (2002).

[19] A. Maksimchuk and V. Yu Bychenkov, Phys. Rev. Lett. 84, 4108 (2000).

[20] E. L. Clark et al., Phys. Rev. Lett. 84, 670 (2000).

[21] E. L. Clark et al., Phys. Rev. Lett. 85, 1654 (2000).

[22] K. Krushelnick, Phys. Plasmas 7, 2055 (2000).

[23] S. P. Hatchett et al., Phys. Plasmas 7, 2076 (2000).

[24] R. A. Snavely et al., Phys. Rev. Lett. 85, 2945 (2000).

[25] H. Habara et al., Phys. Rev. E 69, 036407 (2004).

[26] P. McKenna et al., Phys. Rev. E 70, 036405 (2004).

[27] T. Zh. Esirkepov et al. Phys. Rev. Lett. 92, 175003 (2004).

[28] V. Kumarappan, M. Krishnamurthy and D. Mathur, Phys. Rev. A 66, 033203 (2003).

[29] V. Kumarappan, M. Krishnamurthy and D. Mathur, Phys. Rev. Lett. 87, 085005 (2001).

[30] V. P. Krainov, M.B Smirnov, Phys. Rep. 370, 237 (2002).

[31] H. M. Milchberg, S. J. McNaught and E. Parra, Phys. Rev. E 64, 056402 (2001).

[32] J. Zweiback, T. Ditmire and M. D. Perry, Phys. Rev. A 59, R3166 (1999).

[33] T. Ditmire et al., Nature (London) 398, 489 (1999).

[34] T. Ditmire et al., Nature (London) 386, 54 (1997).

[35] T. Ditmire, R. A. Smith, J. W. G. Tisch and M. H. R. Hutchinson, Phys. Rev. Lett. 78, 3121 (1997).

[36] T. Ditmire et al., Phys. Rev. A 53, 3379 (1996).
[37] Y. L. Shao et al., Phys. Rev. Lett. 773343 (1996).

[38] E. M. Snyder, S. A. Buzza and A. W. Castleman Jr., Phys. Rev. Lett. 773347 (1996).

[39] M. Borghesi et al., Phys. Rev. Lett. 92, 055003 (2004).

[40] M. Borghesi et al., Phys. Rev. Lett. 88, 135002 (2004).

[41] M. Borghesi et al., Phys. Plasmas 9, 2214 (2002).

[42] F. Califano, F. Pegoraro and S. V. Bulanov, Phys. Rev. E 68, 066406 (2003).

[43] S.V. Bulanov et al. Phys. Lett. A 299, 240 (2002).

[44] S.V. Bulanov and V. S. Khoroshkov, Plasma. Phys. Rep. 28, 453 (2002).

[45] T. Zh. Esirkepov et al., Phys. Rev Lett. 89, 175003 (2002).

[46] R. Orecchia et al., Clinical Reviews in Oncology/Hemathology 51, 81 (2004).

[47] A. Brahme, Int. J. Radiat. Oncol. Biol. Phys. 58, 603 (2004).

[48] W. K. Weyrather and J. Debus, Clinical Oncology 15, s23 (2003).

[49] H. Tsujii, Eur. J. Cancer 37, s251 (2004).

[50] R. Orecchia et al., Eur. J. Cancer 34, 459 (1998).

[51] U. Amaldi, analysis 1, 1 (2003).

[52] M. Kanapathipillai et al., Phys. of Plasmas, 11, 3911 (2004).

[53] V. E. Fortov et al., New. J. of Phys. 5, 102 (2003).

[54] J. Kasparian et al., Science 301, 61 (2003). 